\title{
Case Report When Should a Patient with Statin-Induced Myopathy Be Re-challenged? A Case of Necrotizing Autoimmune Myopathy
}

\author{
Elena Obreja $\mathbb{D D}^{1}$ Pamela Sequeira, ${ }^{2}$ and Diana Girnita $\mathbb{D D}^{3}$ \\ ${ }^{1}$ Internal Medicine Resident, Weiss Memorial Hospital, Chicago, IL, USA \\ ${ }^{2}$ Department of Pathology, Trihealth Hospitals, Cincinnati, OH, USA \\ ${ }^{3}$ Department of Rheumatology, Trihealth Physician Partners, Cincinnati, OH, USA \\ Correspondence should be addressed to Diana Girnita; diana_girnita@trihealth.com
}

Received 1 July 2018; Accepted 1 September 2018; Published 1 October 2018

Academic Editor: Mario Salazar-Paramo

Copyright (C) 2018 Elena Obreja et al. This is an open access article distributed under the Creative Commons Attribution License, which permits unrestricted use, distribution, and reproduction in any medium, provided the original work is properly cited.

Statins are notorious for causing myalgia and sometimes mild elevation of CPK (creatine phosphokinase). Herein, we present a case of necrotizing autoimmune myopathy induced by statins. The patient was on therapy with atorvastatin for about six years before she started developing myalgia and mild elevation in CPK that resolved after discontinuation of therapy. Since her cardiovascular risk was high and she had hypercholesterolemia, three months after CPK levels normalization, she was rechallenged with pravastatin. Few months later, she again presented severe myalgia, weakness, and elevated CPK levels. Hence, medication was discontinued, and she undergone an extensive workup for possible causes of inflammatory myopathies that revealed necrotizing autoimmune myopathy. Our case report offers an excellent source of "identification patterns" of muscular autoimmune disease which can be easily mistaken as common side effect of a drug.

\section{Introduction}

Statin-associated myopathy or myalgia is a well-known side effect of lipid-lowering agents. Usually, after discontinuation of the drug, symptoms alleviate and patients may be re-challenged with another statin. However, in rare cases, symptoms of myalgia do not subside after statins are discontinued. Therefore, we are presenting a case report of a rare autoimmune condition that can be induced by statins. We will present the importance of recognition of the clinical manifestations and laboratory changes that can lead to a correct diagnosis. The ultimate educational value of our case report is to highlight the approach in treating these patients and establish when and/or if is safer to expose them to other types of statins.

Necrotizing autoimmune myopathy (NAM) is distinguished from the other inflammatory processes by the acute onset of proximal muscles weakness associated with very high (more than 50 times the upper limit of normal) CPK levels [1]. Therefore, clinical picture, CPK levels, EMG, and MRI muscle are important steps in evaluating a patient with myalgia. However, when suspicion remains, only the muscle biopsy will be the gold standard in clarifying the diagnosis.

\section{Case Presentation}

A 67-year-old African American female with a past medical history significant for type 2 insulin-dependent diabetes mellitus, hypertension, hypercholesterolemia, severe osteoarthritis of the left shoulder, moderate degenerative disc disease of lumbar spine, gout, chronic kidney disease stage III, and chronic pancreatitis was referred to rheumatology with complains of weakness in her upper extremities proximal muscles for about one month. She was on treatment with atorvastatin for hypercholesterolemia for the last 6 years. Due to concern of statin-induced myopathy, the atorvastatin was discontinued a month before her consultation. Around the time of stopping atorvastatin, the patient described flu-like symptoms (low-grade fevers, myalgia, arthralgia, and runny nose). While she was on therapy with atorvastatin, baseline CPK levels were in the $230 \mathrm{mg} / \mathrm{dl}$, with the highest value being $529 \mathrm{mg} / \mathrm{dl}$ at the time of therapy discontinuation (Figure 1).

Her CPK was repeated 20 days later and was increased to $720 \mathrm{mg} / \mathrm{dl}$. The patient was seen in the rheumatology clinic within one week. At the time of her initial evaluation, the 
patient's main complaint was pain in the right shoulder, irradiating to her neck, right wrist, and fingers. Despite her subjective weakness, strength was $5 / 5$ in her proximal and distal upper extremities muscles. She occasionally reported difficulties swallowing and photosensitivity, but denied any lower extremity weakness or difficulties to rise from a chair, rashes, oral/nasal ulcerations, Raynaud's phenomenon, or shortness of breath.

Her physical exam was suggestive of right shoulder impingement syndrome (significantly decreased range of motion, positive Neer's and Hawkins tests, anteroflexion $90^{\circ}$, reduced abduction, adduction, internal rotation, and external rotation), and right wrist examination was significant for mild swelling, limited range of motion, and tenderness to palpation. Left shoulder and wrist examination were unremarkable. Neck flexors and extensors examination was normal. Lower extremities examination revealed $5 / 5$ strength; the patient was able to get out of the chair without pushing herself out.

Routine laboratory studies were significant for normocytic anemia; moderate elevated BUN, CRP of $2.5 \mathrm{mg} / \mathrm{L}$, and CPK levels of $720 \mathrm{mg} / \mathrm{dl}$.

Plain X-rays were obtained, and they were suggestive of severe degenerative osteoarthritis of the right shoulder, chondrocalcinosis of right wrist and knee, and diffuse osteopenia.

Due to her history of gout and CKD, an initial diagnosis of possible crystal induced arthropathy was made.

The patient received a steroid injection in the right shoulder and was started on a short taper of prednisone with complete resolution in her symptoms in two weeks.

At two-month follow-up, she was free of symptoms and CPK levels were normal $(145 \mathrm{mg} / \mathrm{dl})$.

Cardiovascular risk being high and having high cholesterol levels, the decision to re-challenge the patient with another statin was made. This time she was resumed on pravastatin, 3 months after her CPK levels were persistently normal, and she was free of symptoms.

After three months on therapy with pravastatin, the patient experienced recurrent myalgia in proximal muscles of upper but also lower extremities. CPK levels increased again to $586 \mathrm{mg} / \mathrm{dl}$, and the sedimentation rate (ESR) was $51 \mathrm{~mm} / \mathrm{hr}$ (Figure 2). The pravastatin was discontinued.

A myositis panel was obtained and was negative for all antibodies (Table 1). Anti-HMG-CoA reductase antibody was not tested because the patient could not afford the cost of the test.

Therefore, an EMG was performed and revealed peripheral sensory neuropathy but no signs of myopathy. MRI of the right humerus was obtained and showed small bursal effusion, severe osteoarthritis, rotator cuff tear, severe chondral loss, severe tendinosis of subscapularis tendon, and full-thickness tear supraspinatus tendon, but no muscle edema. The patient was referred to neurology to evaluate for muscle weakness.

Since her presentation was not consistent with a neurological disease and her CPK increased further to $1400 \mathrm{mg} / \mathrm{dl}$, we made the decision to obtain muscle biopsy. The pathology report was consistent with inflammatory necrotizing myopathy (intrafascicular inflammation with muscle atrophy, the inflammation is predominantly intrafascicular with actively necrotic muscle fibers) (Figures 3 and 4).

\section{Treatment}

Prednisone $1 \mathrm{mg} / \mathrm{kg} /$ day was initiated with minimal improvement in her symptoms; however; CPK levels started to trend down, but did not normalize after one month on this therapy. Methotrexate was added as a steroid-sparing agent and gradually increased to $20 \mathrm{mg} /$ week over the course of next 2 months. The prednisone was not able to be tapered down due to persistent severe myalgia in her upper and lower extremities as well as elevated levels of CPK (960 IU/L). Intravenous immunoglobulin (IVIG) therapy was initiated (dose: 1 gram for two consecutive days). In one month after first IVIG infusion, CPK levels normalized and the patient experienced significant clinical improvement. The therapy with prednisone, methotrexate, and IVIG will continue.

\section{Discussion}

Assessing serum creatine kinase (CPK) is an important step in evaluating a patient with complains of myalgia or weakness. CPK levels vary according to race, gender, age, and muscle mass. Higher levels were found in men and African Americans. A study done by National Health and Nutrition Examination Survey supported by CDC suggests that the racial differences are not explained by muscle mass but due to differential production or clearance of CPK [2]. The higher median CPK levels in African Americans men are $135 \mathrm{U} / \mathrm{L}$ versus $73 \mathrm{U} / \mathrm{L}$ in African American females, $64 \mathrm{U} / \mathrm{L}$ in white men versus $42 \mathrm{U} / \mathrm{L}$ in white women, and $69 \mathrm{U} / \mathrm{L}$ in Hispanic men versus $48 \mathrm{U} / \mathrm{L}$ in Hispanic women [3]. Therefore, being African American, our patient was at high risk to have higher CPK levels than median.

Among the clues that can lead us to the cause of myalgia associated with elevated CPK is carefully reviewing the medications patients are taking, especially lipid-lowering medication such as statins. Lipid-lowering agents act by inhibiting HMG-CoA reductase, thus reducing cholesterol biosynthesis. Usually statins are well tolerated and less than $0.5 \%$ of the patients' associated clinically significant myonecrosis are manifested by weakness and elevated CPK levels. Statin-associated muscle toxicity includes a wide spectrum of manifestations from simple myalgia (characterized only by muscle pain) to myopathy or myositis (which also associates elevated CPK levels more than 10 times the upper normal limit). Mild muscular symptoms such as myalgia occur in equal percent of patients treated with statins and placebo [4]. In all these cases, symptoms subside after statins are discontinued.

In our case, the initial diagnosis was statin-induced myopathy, so we discontinued statins which resulted in improvement of symptoms and normalization of CPK. However, in the patient's need of lipid-lowering medication, the decision was made to start her on a hydrophilic statin, like pravastatin. The sensitivity to one statin versus another is based on their lipophilic, like atorvastatin, or hydrophilic properties. Lipophilic statins are more toxic than the 


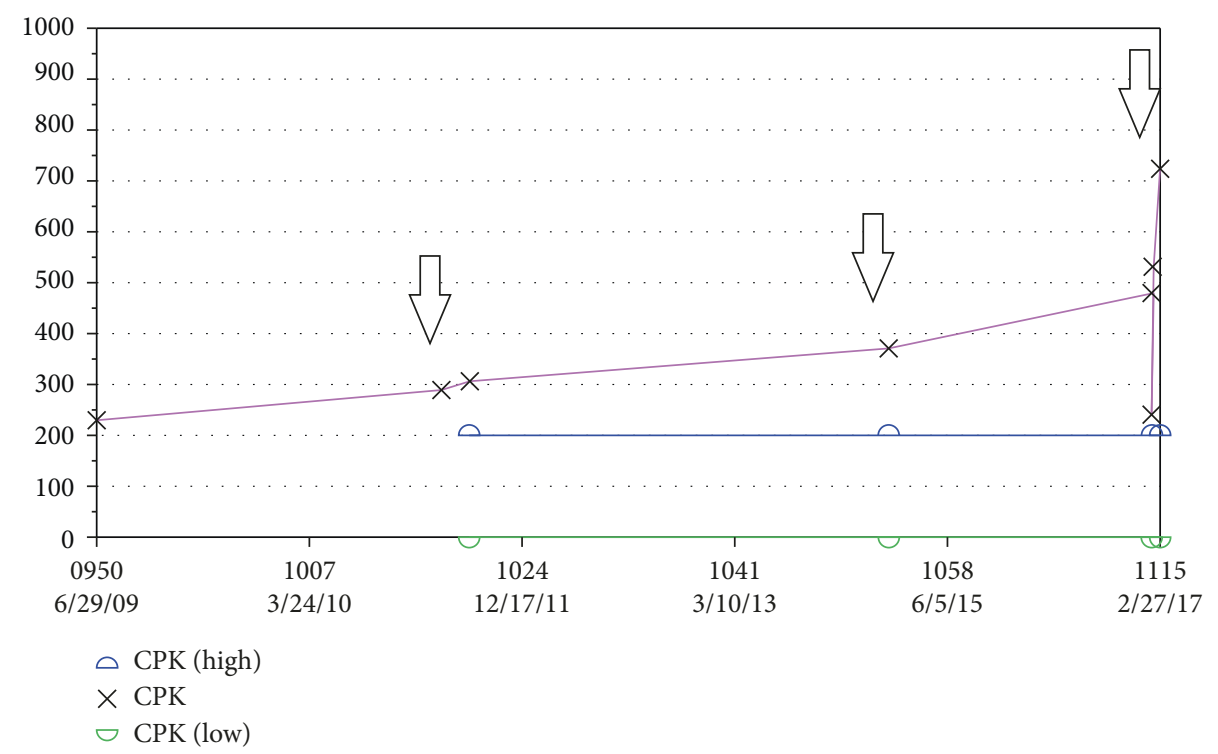

FIGURE 1: CPK levels during statin therapy, before presenting to Rheumatology Clinic.

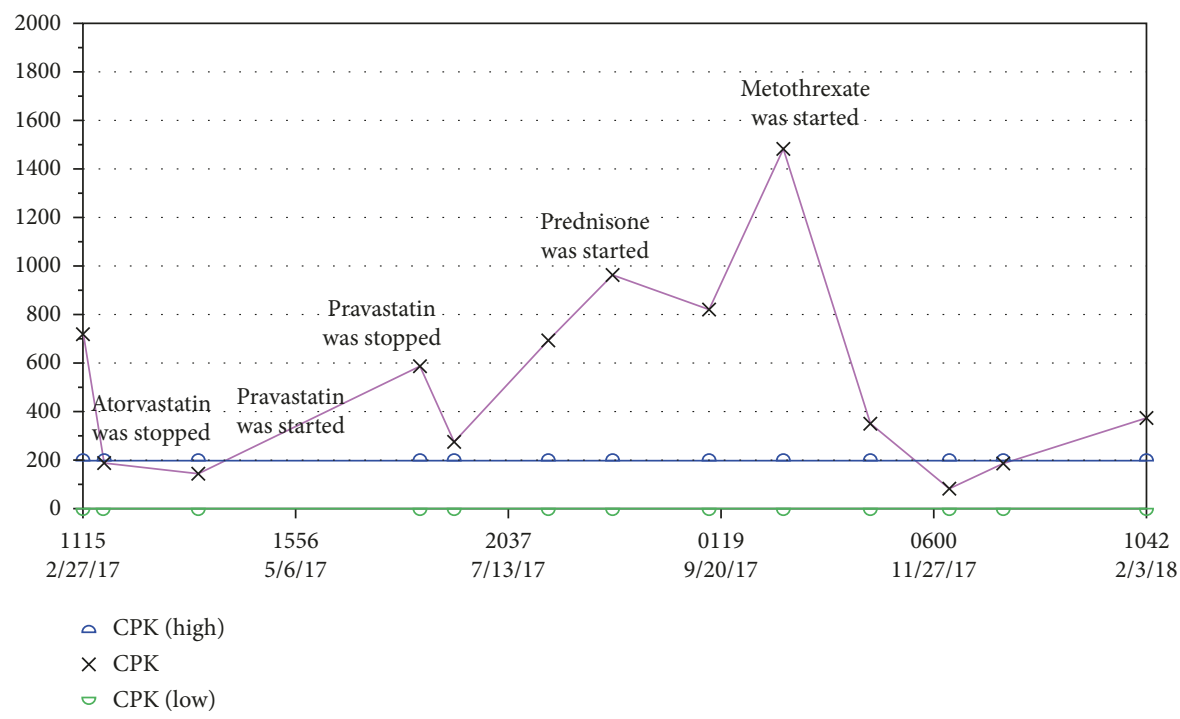

FIGURE 2: CPK levels after statin re-challenge and initiation of immunosuppressive therapy.

hydrophilic ones (rosuvastatin and pravastatin). It is explained by the capacity of the hydrophilic statins to be actively transported in the hepatocytes by expressing organic anionic transporting polypeptide $1 \mathrm{~B} 1$ which regulates hepatic uptake. Thus, lipophilic statins diffuse into nonhepatocyte cells such as myocytes [5]. Furthermore, pravastatin is one of the statins that are not metabolized by CYP3A4 [6]. These mechanisms explain the statin-induced myopathy.

In our case, after re-exposing the patient to a "safer" statin, her myalgia got worse and CPK levels were found to be very high. Therefore, there was a suspicion of inflammatory myopathy and a more detailed workup was done.

Differentiating between the five types of inflammatory myopathies requires a stepwise approach that includes checking the levels of CPK, obtaining EMG, followed by MRI and ultimately performing a muscle biopsy.
In contrast to myalgia or myopathy, autoimmune necrotizing myopathy (NAM) is a very rare condition that is not always associated with statin use, and it accounts for $19 \%$ of all inflammatory myopathies [1]. Statin-associated NAM may also present with dysphagia, arthralgias, or Raynaud's phenomena [7]. There are several other causes of NAM besides the cholesterol-lowering drugs, such as paraneoplastic syndromes (most common types of cancer associated are lung, renal, breast, and ovarian cancer), connective tissue diseases, and HIV infection [8].

EMG in NAM is characterized only by active myopathic units, and the muscle biopsy shows the presence of necrotic fibers, regenerating fibers without significant inflammatory cells, and diffuse or focal upregulation of MHC class I expression [1]. Assessment of autoantibodies can also be helpful but anti-HMG-CoA reductase antibodies are not 
TABLE 1: Myositis panel was negative except for SSA 52 Ab IgG which was equivocal.

\begin{tabular}{lcc}
\hline Antibody & Result & Reference value \\
\hline SSA 52 (Ro) Ab IgG & & $<29 \mathrm{AU} / \mathrm{mL}$ negative, \\
& $32 \mathrm{AU} / \mathrm{mL}$ & 30 AU/mL equivocal, \\
& & $>41 \mathrm{AU} / \mathrm{mL}$ positive \\
SSA 60 (Ro) Ab IgG & $<29 \mathrm{AU} / \mathrm{mL}$ negative \\
Ribonuclei protein U1 Ab IgG & $4 \mathrm{AU} / \mathrm{mL}$ & $<29 \mathrm{AU} / \mathrm{mL}$ negative \\
Jo-1(histidyl-tRNA synthetase) Ab, IgG & $0 \mathrm{AU} / \mathrm{mL}$ & $<29 \mathrm{AU} / \mathrm{mL}$ negative \\
PL-12(alanyl-tRNA synthetase) Ab & $0 \mathrm{AU} / \mathrm{mL}$ & Negative \\
PL-7(threonyl-tRNA synthetase) Ab & Negative & Negative \\
EJ (glycyl-tRNA synthetase) Ab & Negative & Negative \\
OJ (isoleucyl-tRNA synthetase) Ab & Negative & Negative \\
SRP (signal recognition particle) Ab & Negative & Negative \\
Ku Ab & Negative & Negative \\
PM/SCL 100 Ab IgG & Negative & Negative \\
U2 sn (small nuclear) RNP Ab & Negative & Negative \\
Fibrillarin (U3 RNP) Ab, IgG & Negative & Negative \\
Mi-2 (nuclear helicase protein) Ab & Negative & Negative \\
P155/140 Ab & Negative & Negative \\
TIF-1 gamma (155 kDa) Ab & Negative & Negative \\
SAE1 (SUMO activating enzyme) antibody & Negative & Negative \\
MDA5 (CADM-140) Ab & Negative & Negative \\
NXP-2 (nuclear matrix protein-2) Ab & Negative & Negative \\
\hline
\end{tabular}

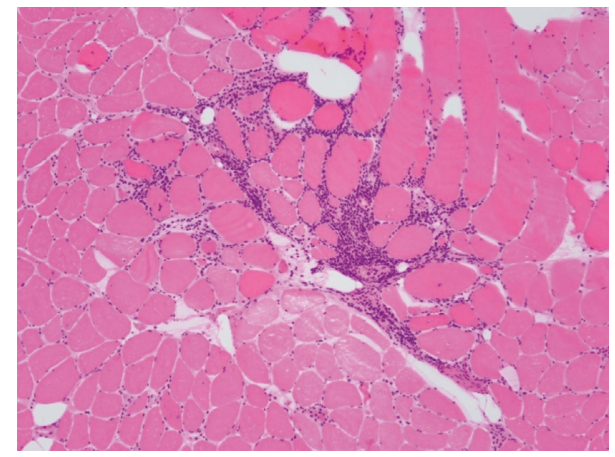

FIGURE 3: Muscle intrafascicular and perivascular lymphocytic inflammation $(\mathrm{H} \& \mathrm{E}$, photomicrograph $\times 100)$.

sensitive and may be present in patients that have not been exposed to statins $[1,9]$.

An importance in the diagnosis of NAM is identification of anti-signal recognition particle autoantibody (SRP) and 3hydroxy-3-methylglutaryl-CoA-reductase (HMGCR) autoantibody [10]. These antibodies have been identified in $60 \%$ of the patients diagnosed with NAM [10]. A study done by Allenbach et al. reported a more severe disease in anti-SRPpositive patients versus anti-HMGCR-positive patients [11]. However, latest reports raise the question that these antibodies might have a possible protective role rather than pathogenic. SRP and HMGCR are involved in proteins synthesis, which can thus explain a regenerating process of the myofibers rather than destruction [10]. Either way, these antibodies can be useful for diagnosing NAM but are not the fundamental piece for diagnosis.

We could only assess the myositis antibodies for our patient since she could not afford to pay for anti-HMG-CoA reductase antibody testing.

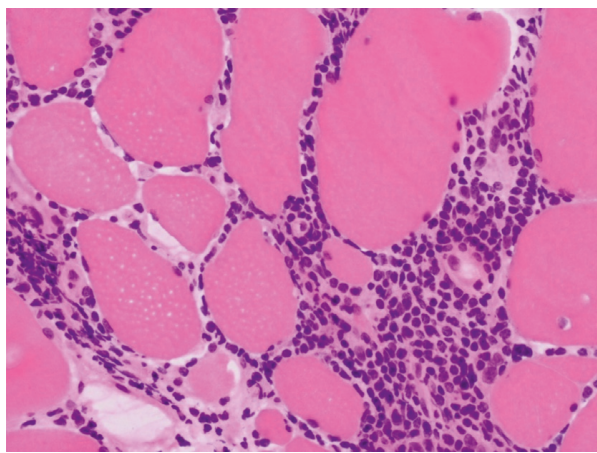

FIGURE 4: Lymphocytic inflammation surrounding muscle fibers (H\&E, photomicrograph $\times 400)$.

A study in 2010 showed that, from all inflammatory muscle disorders, $82 \%$ of the patients with necrotizing myopathy have been previously exposed to statins as compared to the patient with dermatomyositis (18\%), polymyositis (24\%), and inclusion body myositis (38\%) [12].

Cancer screening was also performed in our case. Colonoscopy, mammogram, and CT chest/abdomen/pelvis were all negative.

The mechanism by which statins can cause autoimmune necrotizing myopathy is supposed to be due to overexpression of HMG-CoA reductase in the majority of patients with class II HLA allele DRB1 $* 11: 01$ [9]. Studies have shown that people with this allele develop anti-HMG-CoA reductase antibodies. This is observed even in patients who have not been exposed to statins. The expression of HMGCoA reductase is markedly increased when cells are exposed to statins and also in regenerating myocytes. Also, the premise is that $\mathrm{MCH}-\mathrm{I}$ is upregulated, and statins induce an autoimmune myopathy [5]. All these suggest that statin- 
induced NAM is a distinct pathology from self-limiting statin-induced myopathy.

Being a rare entity, there are not many case reports that can differentiate the autoimmune response in patients taking hydrophilic versus lipophilic statins. Either way, as we could prove, even re-challenging patients with a "safer" type of statin does not have a benefit with regard to the muscular symptoms. Statins should be discontinued, and immunosuppressive therapy should be started to achieve amelioration of the patient's symptoms.

Treatment needs to be aggressive and initiated as soon as the diagnosis is confirmed. Steroids, methotrexate, and in severe cases IVIG can change the prognosis in this disease. IVIG therapy was initially introduced for the treatment of primary immunodeficiency diseases, but subsequently it has been used to target autoimmunity as well. The mechanism by which it exerts anti-inflammatory effects is still not fully understood, but it is known that the doses for treating inflammatory conditions are four to five times higher than for those used for replacement [13].

Administration of IVIG helps improving autoimmunity process by different mechanisms such as decreasing cytokines levels, blocking the inflammation, and decreasing IgG saturation, thus increasing IgG levels in circulation [13].

Only after one infusion of IVIG, our patient's symptoms have markedly improved and her CPK levels went back to normal, proving to be a successful approach.

\section{Conclusion}

Our case report reveals a rare entity, NAM often mistaken as statin-induced myalgia. The first instinct was to discontinue the offending drug to alleviate. Most often, this is the solution. However, after re-exposing the patient to a different statin that will cause recurrence of symptoms, we encourage the physicians to obtain a more detailed workup as that could change the prognosis of the patient's disease.

\section{Conflicts of Interest}

The authors declare that there are no conflicts of interest regarding the publication of this paper.

\section{References}

[1] M. C. Dalakas, "Inflammatory muscle diseases," New England Journal of Medicine, vol. 372, no. 18, pp. 1734-1747, 2015.

[2] M. D. George, N.-K. McGill, and J. F. Baker, "Creatine kinase in the U.S. population," Medicine (Baltimore), vol. 95, no. 33, p. e4344, 2016.

[3] R. Neal, "Relationship of ethnic origin, gender, and age to blood creatine kinase levels," American Journal of Medicine, vol. 122, no. 1, pp. 73-78, 2009.

[4] T. T. Abd, "Statin-induced myopathy: a review and update," Exoert Opinion on Drug Safety, vol. 10, no. 3, pp. 373-387, 2011.

[5] R. Bitzur, H. Cohen, Y. Kamari, and D. Harats, "Intolerance to statins: mechanisms and management," Diabetes Care, vol. 36, no. 2, pp. S325-S330, 2013.
[6] R. Rosenson, Statin Muscle-Related Adverse Events, UpToDate, Waltham, MD, USA, 2018, https://www.uptodate.com/ contents/statin-muscle-related-adverse-events\#H19.

[7] J. Albayda and L. Christopher-Stine, "Identifying statinassociated autoimmune necrotizing myopathy," Cleveland Clinic Journal of Medicine, vol. 81, no. 12, pp. 736-741, 2014.

[8] Y. Allenbach, J. Keraen, A.-M. Bouvier et al., "High risk of cancer in autoimmune necrotizing myopathies: usefulness of myositis specific antibody," Brain A Journal Of Neurology, vol. 139, no. 8, pp. 2131-2135, 2016.

[9] A. L. Mammen, "Statin-associated autoimmune myopathy," New England Journal of Medicine, vol. 347, no. 7, pp. 664-669, 2016.

[10] M. C. Dalakas, "Are autoantibodies pathogenic in necrotizing myopathy?," Nature Reviews Rheumatology, vol. 14, no. 5, pp. 251-252, 2018.

[11] Y. Allenbach, L. Arouche-Delaperche, C. Preusse et al., "Necrosis in anti-SRP+ and anti-HMGCR+myopathies: role of autoantibodies and complement," Neurology, vol. 90, no. 6, pp. e507-e517, 2018.

[12] P. Grable-Esposito, H. D. Katzberg, S. A. Greenberg, J. Srinivasan, J. Katz, and A. A. Amato, "Immune-mediated necrotizing myopathy associated with statins," Muscle Nerve, vol. 41, no. 2, 2009.

[13] E. Gelfand, "Intravenous immune globulin in autoimmune and inflammatory diseases," New England Journal of Medicine, vol. 367, no. 21, p. 2015, 2012. 


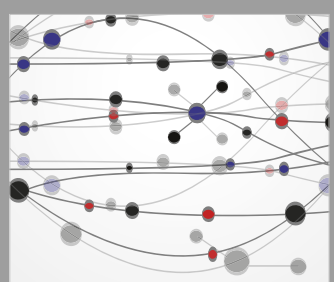

The Scientific World Journal
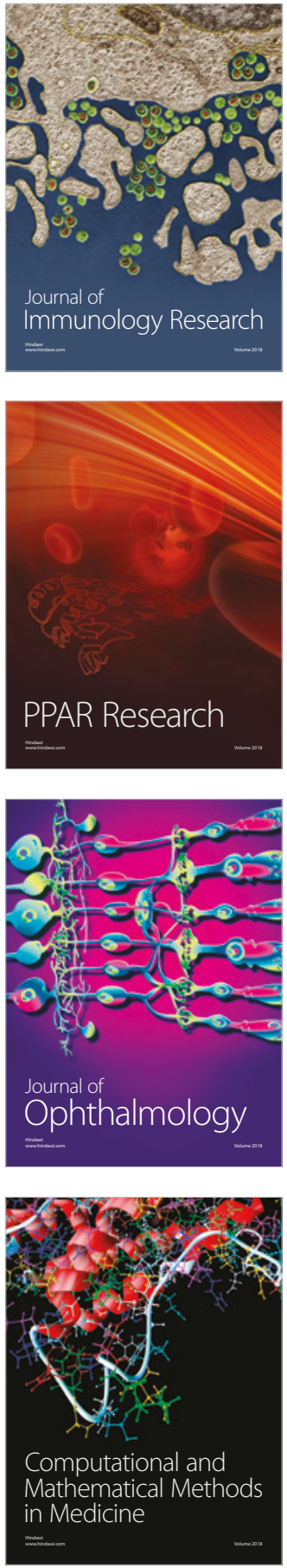

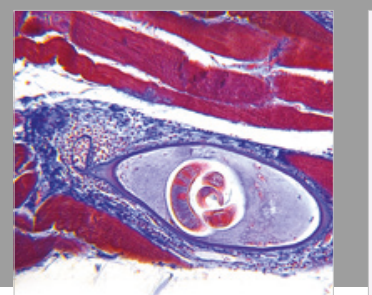

Gastroenterology Research and Practice

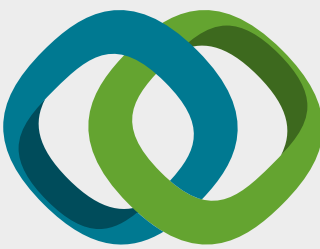

\section{Hindawi}

Submit your manuscripts at

www.hindawi.com
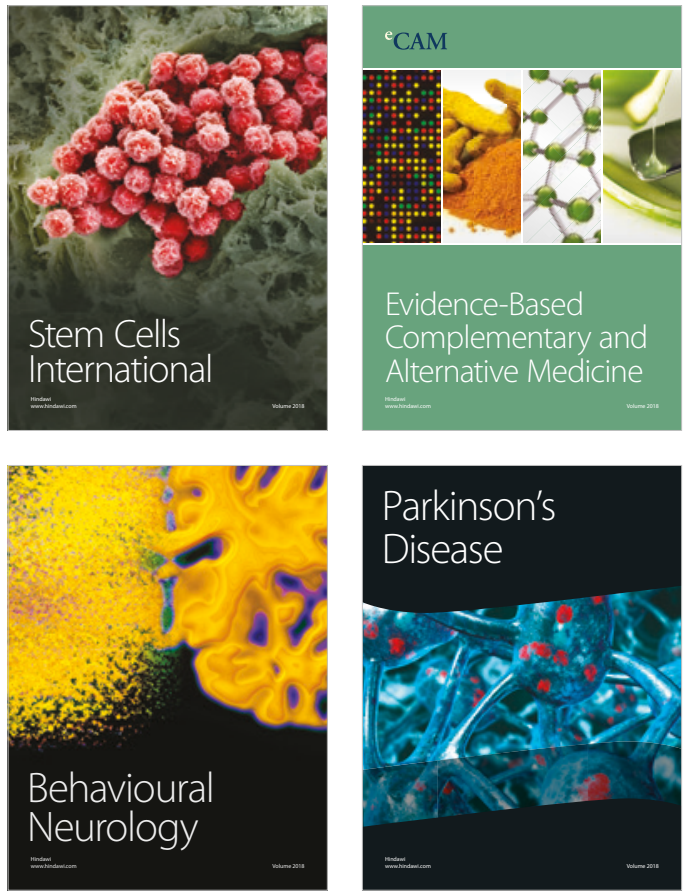

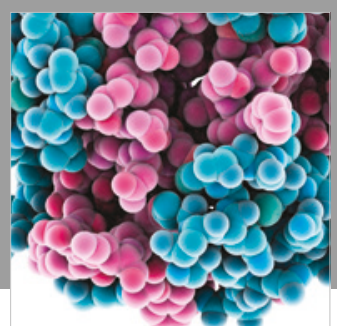

ournal of

Diabetes Research

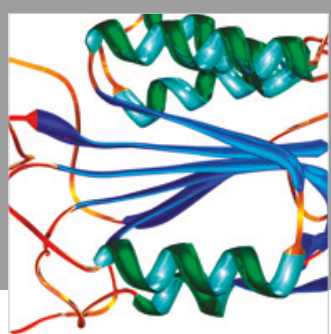

Disease Markers
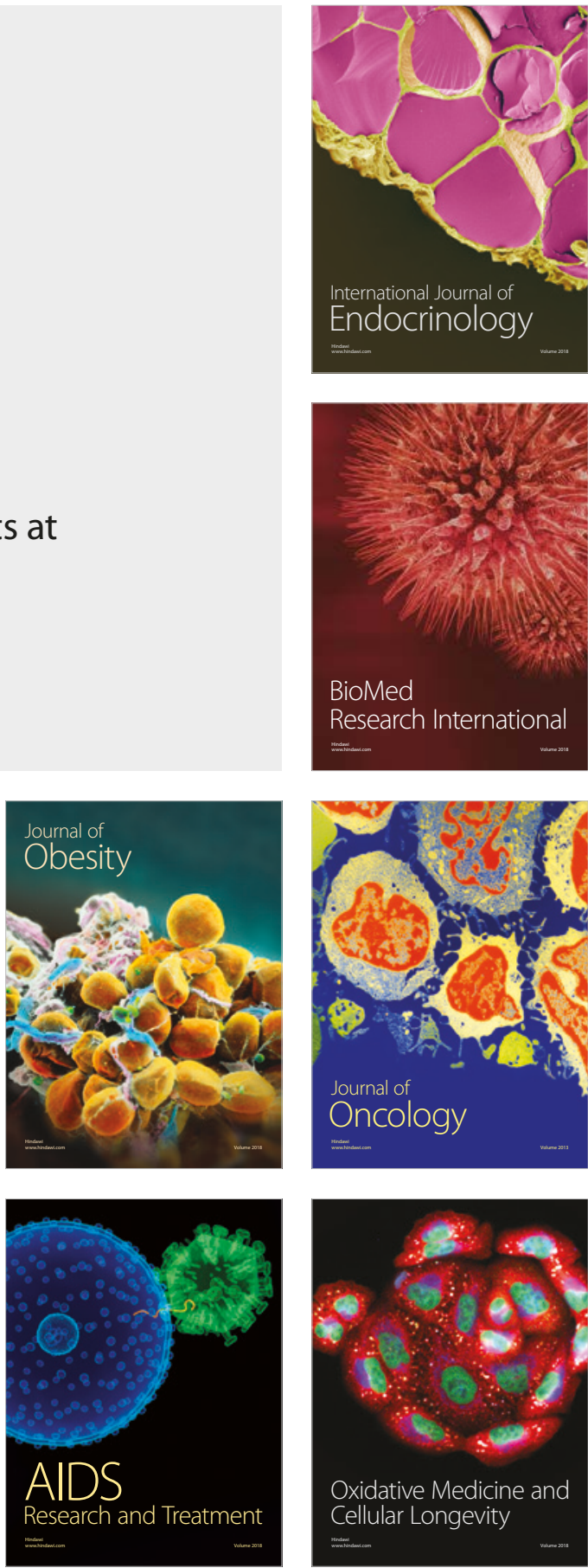